\title{
THE QUESTIONS OF DEVELOPMENT OF FIBER- OPTIC SENSORS FOR MEASURING PRESSURE WITH IMPROVED METROLOGICAL AND OPERATION-AL CHARACTERISTICS
}

\author{
Alexey Yurchenko ${ }^{1,3,}$, , Aliya Alkina ${ }^{1}$, Ali Mekhtiev $^{2}$, Felikc Bulatbayev $^{2}$, Elena Neshina $^{2}$ \\ ${ }^{1}$ National Research Tomsk Polytechnic University, 634050, Tomsk, Russia \\ ${ }^{2}$ Karaganda State Technical University, 100027, Karaganda, Kazakhstan \\ ${ }^{3}$ National Research Tomsk State University, 634050, Tomsk, Russia
}

\begin{abstract}
One of the promising directions of FOPS implementation is the use them as the new generation sensors of information-measuring systems for measuring the pressure in pneumatic system of mining machinery. The use of FOPS will allow you to solve a number of security issues when operating in difficult and dangerous conditions of the mining industry. In this paper we have developed an experimental model that allows obtaining a high level of sensitivity due to the intensity of the output emitting modulation. Based on the results of experimental researches of FOPS sample the functional dependences are constructed confirming its efficiency of work. The implementation of FOPS will provide the high precision of pressure parameters measurements compared to mechanical and electronic systems and in this regard it is provided the high reliability of its operation in hazardous conditions of mining industry.
\end{abstract}

\section{Introduction}

The modern fiber-optic sensors (FOS) are used for measuring virtually all physical quantities. For example, they can determine with high precision the value of pressure and temperature, position in space, speed of rotation and linear displacement, acceleration, torque, twisting, oscillation, vibration, weight, pressure of sound wave, liquid level, strain, index of refraction, electric field intensity, magnitude of electric current and magnetic field, concentration of gas and various contaminants, the dose of radiation, etc. [1]

The basic elements of the fiber-optic sensor are the optical fiber, light-emitting (light sources) and light-receiving devices, the optical sensing element [1]. The demand for sensors with similar characteristics soars in areas such as mining, aerospace and defense industry, materials production, medicine and construction. Their use will create inside object fiber-optic information systems of parameters and physical quantities measurements and of performance equipment diagnostics. Especially these issues are relevant to mining machinery as their technical condition monitoring will avoid serious accidents and even any

Corresponding author: niipp@inbox.ru 
loss of life and so the use of optical fiber allows obtaining a number of significant advantages compared to traditional electronic measuring systems especially under conditions of spark- and explosion hazards at production sites where there is a danger of gas explosion and dust.

Considering all the advantages one may single out the most important fact that at the pre-sent time in Kazakhstan it is practically not carried out the works on creation of information- measuring and diagnostic systems (IDS) based on FOS and existing achievements are limited to theoretical works. The significant gap in the field of FOS from foreign ones can be explained by the lack of a systematic and integrated approach to solving this problem as well as insufficient financing of this area due to the underestimation of the capabilities of FOS. Currently, in the mining industry there is a need for the Next Generation IDS with improved parameters [2].

The analysis of scientific and technical literature showed that the questions of using it in the mining industry are weakly reflected. The use of fiber-optic pressure sensors (FOPS) will allow you to solve a number of security issues when operating in difficult and dangerous conditions of the mining industry. For the great majority of developed both domestic and foreign FOPS it is used the design connected with reflection effect. It is a relatively simple concept but at the same time it has a number of disadvantages associated with the fact that a part of light emission is lost when reflected from the membrane which leads to decrease in sensor sensitivity and measurement accuracy. The achievement of reducing the loss of light in FOPS especially in the area of measured value will improve the accuracy of the measurement [2].

In view of this the aim of our work is the development of FOPS with improved metrological and operational characteristics, with simplified design so it can be implemented in mining enterprises. One of the promising directions of implementation of FOPS is the use them as the next generation sensors of information-measuring systems for measuring the pressure in pneumatic systems of mining machinery. The precise control of pressure parameters is an important factor in ensuring the regulatory safety requirements in hazardous production and it is required to provide the spark- safe circuit [3].

\section{The fiber-optic pressure sensors}

We have developed an experimental model of FOPS (Figure 1) in accordance with the tasks as-signed that contains an optical fiber (single-mode) 1 with core 2 as the measuring element. It is installed in the housing 3 with the sawn groove for placing the optical fiber light guide 1 . The optical fiber light guide is polished on the part of its length to formation of a flat chamfer 4 . In this case the membrane 5 with protrusion 6 is fixed to the housing 3 . The liquid 7 is placed be-tween the membrane with the protrusion 6 and the housing 3 .

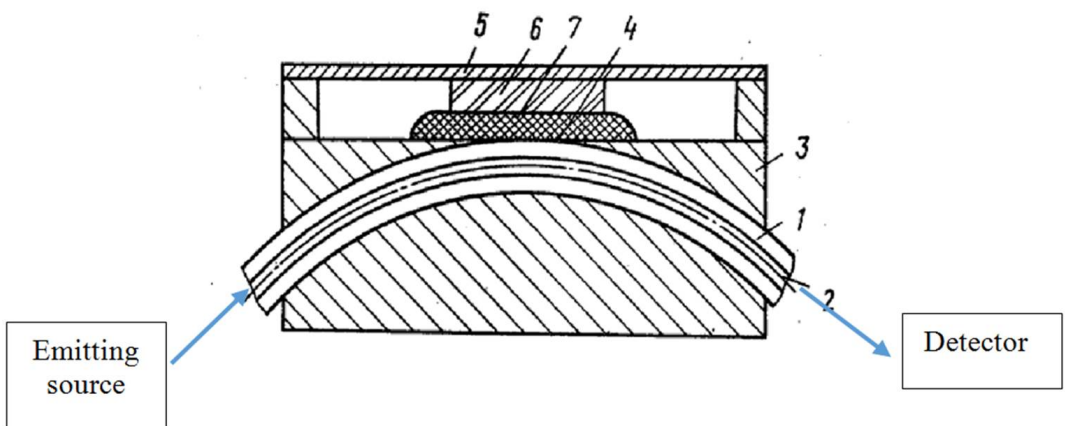

Figure 1. FOPS construction. 
This construction allows you to obtain a high level of sensitivity due to the intensity of modulation of the output emitting resulting from violation of mode and fluid locking arising from the displacement of the membrane in the range of $0.01 \mu \mathrm{m}$. In FOPS it is connected to a source of emitting of optical range. With a change of pressure there is the pressure on the mem-brane 5 causing its movement. This leads to a change in distance between the protrusion 6 and the chamfer 4 located on optical fiber and this results in the change in modes synchronization in the liquid 7. It is necessary to achieve the equality of modes of optical fiber and liquid wave-guide at the place of connection where it occurs the transfer of radiation power from the fiber to the liquid light guide, the phase synchronism is achieved in its thickness and determined by the following ratio, when $\Delta \beta=0$ : In the optical fiber light guide 1 it is administered

$$
K_{2}^{2}=\frac{2 \pi}{\lambda} \cdot n_{2}^{2}
$$

Where $n_{1}$ is the refractive index of cladding; $n_{2}$ is the refractive index of liquid

$$
K_{3}^{2}=\frac{2 \pi}{\lambda} \cdot n_{3}^{2}
$$

Where $n_{3}$ is the refractive index of the polished plate (of membranes protrusion) $m=0,1,2 \ldots-$ integral nonnegative number.

The change in the constants of the modes distribution of the liquid waveguide when moving the protrusion 6 of the membrane leads to a violation of the condition of phase synchronism of modes of the optical fiber light guide and liquid waveguide that causes a change in the value of branch line from the optical light guide in liquid waveguide that causes a decrease in power of the optical radiation in output of the optical fiber light guide. The equality of the constants (phase synchronism) of the waveguide modes is achieved when the thickness of the liquid waveguide is defined with the following ratio, when $\Delta \beta=0$

$$
t=\frac{1}{\left(K_{2}^{2}-\beta^{2}\right)^{1 / 2}}\left[\operatorname{arctg} \frac{\left(\beta^{2}-K_{1}^{2}\right)^{1 / 2}}{\left(K_{2}^{2}-\beta^{2}\right)^{1 / 2}}+\operatorname{arctg} \frac{\left(\beta^{2}-K_{3}^{2}\right)^{1 / 2}}{\left(K_{2}^{2}-\beta^{2}\right)^{1 / 2}}+m \pi\right]
$$

Where $\beta=\beta_{0}+\Delta \beta$

$\beta_{0}$ is the constant of distribution of single-mode optical fiber light guide;

$$
\Delta \beta=\frac{\sqrt{3}}{2} \cdot K
$$

$K$ is the coupling value between fiber and liquid waveguides;

$$
K_{1}^{2}=\frac{2 \pi}{\lambda} \cdot n_{1}^{2}
$$

$\lambda$ - wavelength of light emission;

When the membrane (protrusion 6) is displaced there is a change in the distribution of the constant modes of the liquid waveguide 7 and it creates the conditions in which the phase synchronism of modes of the light guides 1 and 7 is broken that increases the amount of additional emitting losses and changes the level of scattering in the light guide. To ensure the high sensitivity of the sensor it is required to branch off $50 \%$ of the total power of optical radiation to the liquid light guide. Our sensor is produced on the basis of singlemode fiber made of quartz with cladding diameter of $125 \mu \mathrm{m}$ and core diameter of $9 \mu \mathrm{m}$. The refractive indexes of the core are 1.462 and 1,456 of cladding. The base is made of quartz in which there is the sawed notch with width of $150 \mu \mathrm{m}$ and radius of $200 \mathrm{~mm}$ [4]. Glycerin is used as the liquid light guide. When there is a displacement of the membrane 
protrusion at $0.01 \mu \mathrm{m}$ it is achieved $50 \%$ modulation of the op-tical emission passing through the optical fiber light guide 1 , as the modulation is based on the process of violation of phase synchronism of the modes and the high sensitivity of the sensor is achieved [5]. It is used the semiconductor laser (light-emitting diode) as an emitting source and the optical power meter (OM3-65) as the receiver.

The results of experimental studies were used while constructing the approximating functions, analytical dependence of the output power of optical emission from the applied load is represented as follows:

a) mathematical model of the 4th degree:

$$
-0.0000524476 m^{4}+0.00116162 m^{3}-0.00966783 m^{2}-0.00640637 m+1.00175=f\left(P_{\text {out }}\right) \text {; }
$$

b) quadratic approximation (quadratic model):

$$
-0.00142191 m^{2}-0.0259627 m+1.00958=f\left(P_{\text {out }}\right) \text {; }
$$

c) cubic approximation (cubic model):

$$
0.000112665 m^{3}+0.00311189 m^{2}-0.0195183 m+1.00552=f\left(P_{\text {out }}\right)
$$

On the data obtained the analysis of the developed models on the criterion of Akaike (AIC) was carried out which showed that the most accurate one will be the cube model because it has the smallest criterion value [6]. The boundary conditions of the mathematical model are the load on FOPS from 0 to $10 \mathrm{~kg}$, ambient temperature is $+22^{\circ} \mathrm{C}$, relative humidity of the air is up to $60 \%$; atmospheric pressure is $102 \mathrm{kPa}$. Initial conditions are $P_{\text {out }}=0 ; m=0$.

The dependency graph of the output power from the applied load is presented in Figure 2. The researches have shown that the developed FOPS has a negligible nonlinearity when the load is equal to one kilogram and the stable linearity of characteristics is remained while increasing the load from 2 to $10 \mathrm{~kg}$.

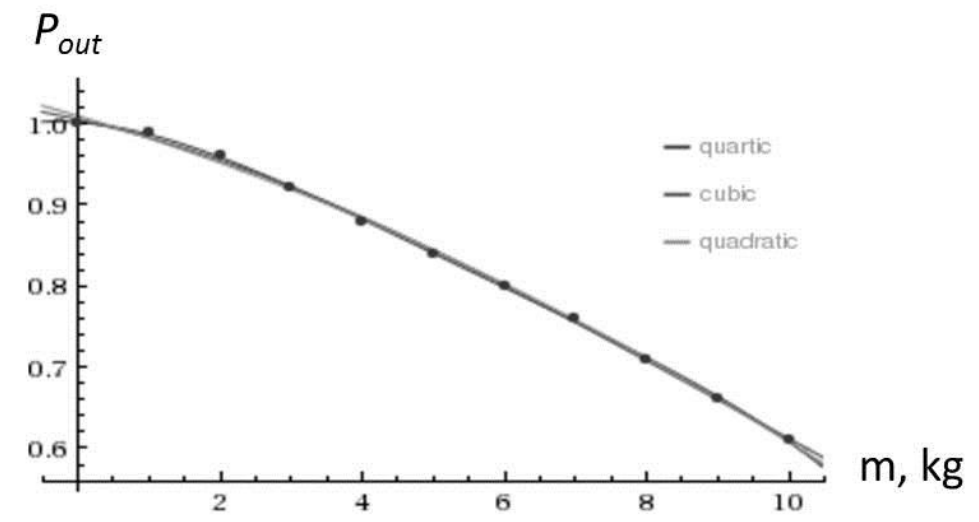

Figure 2. The dependency graph of the output power from the applied load.

\section{Conclusion}

The conclusion is that the use of FOPS will provide the high accuracy of measurement of pressure parameters with the provision of safety requirements. The obtained results allow 
us to adapt FOPS for measuring the parameters of the pneumatic system of the brake device of the mine winder.

\section{Acknowledgements}

The paper was written as part of the research project No. 8.2.31.2015, carried out with the support of the Program "Research Foundation of Tomsk State University named after D.I. Mendeleev" in 2015 - 2016., grant RFBR No. 16-29-12858.

\section{References}

[1] A.D. Mehtiyev, E.G. Neshina, A.P. Bilichenko, Collection of XIX all-Russian scientific-technical conference "Modern problems of radio electronics", 101 (2016)

[2] A.V. Mitrofanov, D.A. Sidorov-Biryukov, A.A. Voronin, A. Pugžlys, A.A. Lanin, A.B. Fedotov, V.Ya. Panchenko, A. Baltuška, A.M. Zheltikov, Optics and Spectroscopy 119, 569 (2015) doi: 10.1134/S0030400X15100185

[3] J. Elazar, S. ${ }^{`}$ Selmi, M. Tomic, M. Prokin, J. Opt. A: Pure Appl. Opt.4, S347 (2002) doi: $10.1088 / 1464-4258 / 4 / 6 / 379$

[4] A. D. Mehtiyev, A. V. Yurchenko, N. I. Gorlov, A.A. Kovtun, Journal of Physics: Conference Series 671, 012001 (2016) doi: 10.1088/1742-6596/671/1/012001

[5] N.R. Krutyak, D.A. Spassky, I.A. Tupitsyna, A.M. Dubovik, Optics and Spectroscopy 121, 45 (2016) doi: 10.1134/S0030400X16070122

[6] D.O. Zyatkov, A.V. Yurchenko, V.B. Balashov, V.I. Yurchenko, IOP Science. Journal of Physics: Conference Series 671, 012065 (2016) doi :10.1088/1742$6596 / 671 / 1 / 012065$ 